\title{
Coherent regulation in Yeast's cell-cycle network
}

\author{
Neşe Aral $^{1}$ and Alkan Kabakçıŏlu ${ }^{1}$ \\ ${ }^{1}$ Department of Physics, Koç University, Rumelifeneri Yolu Saryer 34450, Istanbul, Turkey
}

\begin{abstract}
We define a measure of coherent activity for gene regulatory networks, a property that reflects the unity of purpose between the regulatory agents with a common target. We propose that such harmonious regulatory action is desirable under a demand for energy efficiency and may be selected for under evolutionary pressures. We consider two recent models of the cell-cycle regulatory network of the yeast, Saccharomyces cerevisiae as a case study and calculate their degree of coherence. A comparison with random networks of similar size and composition reveals that the yeast's cell-cycle regulation is wired to yield and exceptionally high level of coherent regulatory activity. We also investigate the mean degree of coherence as a function of the network size, connectivity and the fraction of repressory/activatory interactions.
\end{abstract}

\section{INTRODUCTION}

Despite vast amount of data on genetic regulatory interactions in a growing number of organisms [1 6], the interplay between structure and function in these systems is still unclear and an active field of research [7 9]. Much effort has been devoted to local structures and their role in promoting multistationarity, stability and robustness in a background of fluctuating environmental conditions [10 13]. Other studies argue that regulatory dynamics is "consistent" in the sense that, a transcription factor's (TF) influence on a target gene (activation/inhibition) is not context dependent [14]. Similarly, architectural features allowing better controllability have been of recent interest [15-17]. Such guiding principles provide valuable insight and a bird's-eye perspective on networks of gene regulatory interactions, where a qualitative understanding of the underlying generative mechanisms is hindered by the prohibitive complexity of the cell and the shear amount of experimental data.

We here introduce the concept of "coherent regulation" as a similar guideline for the evolutionary design of regulatory networks. Below, we give a mathematical definition for the degree of coherence, as a measure of the extent to which a given regulatory network structure and the equations of motion for the regulation dynamics are optimized towards minimum waste of material/energy resources available to the cell. We then calculate it for the yeast cell-cycle network, a well-studied subnetwork responsible for cell-division in the yeast Saccharomyces cerevisiae [18], by means of two 
previously proposed models in the literature. Further analyses on random graphs yield a reference baseline as a function of the network's structural parameters, by means of which we show that the yeast's cell-cycle network is, in fact, optimally organized to maximize coherent regulatory action.

\section{MODEL}

\section{A. Coherent regulation}

Regulation of gene activity requires production of regulatory proteins which demands energy and raw material. The need for the economical use of these resources has been a constant and dominant evolutionary pressure in much of the last 4 billion years and virtually for all species [19] 21. Therefore, it is natural to expect regulatory networks to have been optimized towards their optimal use.

A full-fetched analysis of such optimization would require a much deeper understanding than we currently have of a cell's functions on the protein level. Here, we will discuss network efficiency only in terms of the choice of interactions that determine the time dependence of protein expression, and assert that a prerequisite for optimality is to avoid simultaneous production of regulatory elements that perform opposing tasks. In particular, it is desirable that in a steady state, two TFs where one is an activator and the other is a repressor of the same gene are not co-expressed. Exceptions are the relatively brief transition periods (induced by internal or environmental conditions) between steady states or between the states in cyclic attractors (such as circadian cycles [22, 23]), where changes in the expression levels are due to role switches between such antagonistic regulators.

We coin the concordance of simultaneously active regulatory elements sharing a common target as "coherent regulation". More precisely, we say that a gene is coherently regulated if its expression state is determined unanimously by its active regulatory partners, as described in Fig. 1. Note that, coherence defined as above is different from "cooperativity" in regulatory networks discussed in the literature [24], which refers to increase (or decrease) of the binding affinity of a TF when other TFs are already bound to the promoter region of the gene. It can also be noted that, a coherent network as defined above is expected to be more robust against fluctuations in the expression rates of the co-regulating elements. This is because, when the regulatory inputs received by a gene are compatible, fluctuations in their relative strengths (e.g., due to time delays) are less significant than when the regulatory agents act antagonistically, in which case the fluctuations may result in a sign change in the net regulatory message received by the target gene. 
The degree of coherent regulation is clearly structure dependent, i.e., some networks are more supportive of such harmonious regulatory activity than others. Identifying the architectural aspects of these networks is an interesting problem in itself which shall be addressed in a future study. The efficiency acquired through coherence also has a truly dynamical aspect, since coherent action is reached through the time-dependent interplay between expression levels of communicating genes. Starting from the fact that the structure shapes regulatory dynamics in a phenotype, the question we ask here is whether evolutionary processes may have provided the means to feed information from the dynamics back to the structure, promoting modifications that maximize coherent regulatory action.

\section{B. Quantifying coherence in regulatory networks}

Given a fixed environment, the genes/proteins that participate in a genetic regulatory network spend most of their time in the attractors of the regulation dynamics. Let $\tau(\boldsymbol{\sigma})$ be the appropriate discrete-time-evolution operator $\tau$ that propagates the expression level $\boldsymbol{\sigma}$ of the involved TFs from time $t$ to $t+\delta t$, i.e., $\boldsymbol{\sigma}(t+\delta t)=\tau[\boldsymbol{\sigma}(t)]$. Then the attractors are determined by the condition $\boldsymbol{\sigma}^{*}=\tau^{q}\left[\boldsymbol{\sigma}^{*}\right]$. A point attractor corresponds to $q=1$. Otherwise, the attractor is a cycle of period $q \delta t$ with the cycle states $\left\{\boldsymbol{\sigma}^{*}, \tau\left[\boldsymbol{\sigma}^{*}\right], \ldots, \tau^{q-1}\left[\boldsymbol{\sigma}^{*}\right]\right\}$. We here consider Boolean models where $\boldsymbol{\sigma}$ is a binary vector with entries $\in\{0,1\}$.

For stochastic systems, this formulation is easily generalized with, $\tau \rightarrow T$, a transition matrix, and $\boldsymbol{\sigma} \rightarrow \boldsymbol{\pi}$, the probability distribution on the ensemble of states $\{\boldsymbol{\sigma}\}$. While noise is a relevant determinant of cell activity [25-27], biological systems are typically robust to fluctuations in

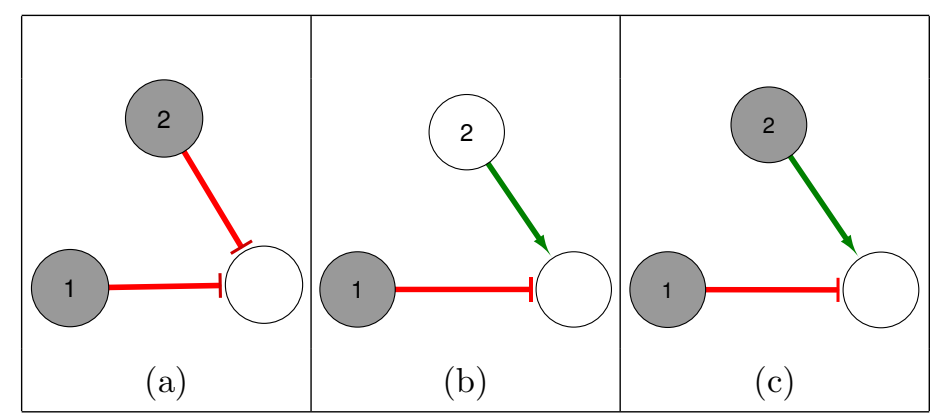

FIG. 1: Coherent vs incoherent regulation for a gene with two regulating partners. Active nodes are shown gray. The target gene is (a) coherently regulated when the two active regulators act unanimously; (b) also coherently regulated when the two antagonistic regulators are not active at the same time; (c) incoherently regulated when antogonistic regulators are simultaneously expressed. 
gene expression levels [28, 29]. Therefore, both stochastic and deterministic models of regulatory dynamics are frequently used in the literature [18, 30, 31].

We can now define the "coefficient of coherence" for an attractor $i$ as $\alpha_{c}^{(i)}$, the fraction of genes which are not subject to simultaneous and conflicting regulatory messages from their regulating partners active in state $i$. Describing up-(down-) regulation of gene $j$ by the associated TFs $k_{j}$ as $c_{j k_{j}}=1(-1)$, we can express $\alpha_{c}^{i}$ in terms of (Boolean) expression levels as:

$$
\alpha_{c}^{i}=\frac{1}{N} \sum_{j=1}^{N} i n t\left(\frac{\left|\sum_{k_{j}} c_{j k_{j}} \boldsymbol{\sigma}_{k_{j}}^{(i)}\right|}{\sum_{k_{j}}\left|c_{j k_{j}}\right| \boldsymbol{\sigma}_{k_{j}}^{(i)}}\right)
$$

Above, $N$ is the number of genes in the network. $i$ is the attractor index, hence for a network with $n_{a}$ attractors $i$ runs from 1 to $n_{a}$. int() function returns the integer part of its argument, which effectively sets the coefficient of coherence to unity if the currently expressed subset of regulators $\left\{k_{j}\right\}$ of gene $j$ are all activators or all repressors, and to zero otherwise. For a cyclic attractor with period $q_{i} \delta t, \alpha_{c}^{i}$ is taken as the arithmetic average over the cycle states $\boldsymbol{\sigma}_{k}^{(i)}\left(k=1, . ., q_{i}\right)$. The coefficient of coherence for a network is defined to be the mean of $\alpha_{c}^{i}$ over all the attractors $i$, i.e.,

$$
\alpha_{c}=\frac{1}{n_{a}} \sum_{i=1}^{n_{a}} \alpha_{c}^{(i)}
$$

where, again, $n_{a}$ is the number of attractors. Alternatively, one could consider the average weighted by the basin sizes (the fraction of uniformly randomly picked initial states that end up in a given attractor):

$$
\alpha_{c}=\frac{1}{\sum_{i=1}^{n_{a}} b_{i}} \sum_{i=1}^{n_{a}} b_{i} \alpha_{c}^{(i)},
$$

where $b_{i}$ is the number of initial states reaching attractor $i$ under the given dynamics. However, such weighted averaging should be employed with caution, because a randomly constructed expression state will typically never appear throughout the life cycle of a cell and, therefore, is irrelevent in the biological sense. We here present our results with both basin-weighted and uniformly weighted averages, in order to demonstrate that our conclusions do not critically depend on this choice.

Finally, the definition given above can also be generalized to continuous models of gene expression by replacing the averages over states with time averages and employing an appropriate extension of the above coherence measure to continuous variables. For simple linearized models of the form

$$
\frac{d n_{j}(t)}{d t}=\sum_{k_{j}} \gamma_{j k_{j}} n_{k_{j}}(t)
$$


where $n_{j}$ typically represent protein/RNA concentrations, a possible generalization could be to replace equation (1) with

$$
\alpha_{c}^{i}=\frac{1}{T_{i} N} \sum_{j=1}^{N} \int_{0}^{T_{i}} d t \frac{\left|\sum_{k_{j}} \gamma_{j k_{j}} n_{k_{j}}(t)\right|}{\sum_{k_{j}}\left|\gamma_{j k_{j}}\right| n_{k_{j}}(t)}
$$

where $T_{i}$ is the period of the $i$-th dynamical attractor in consideration.

\section{RESULTS}

\section{A. Yeast's cell-cycle: a case study}

The budding yeast, Saccharomyces cerevisiae, is a well-studied single-cell eukaryote [2, 32, 34]. Its 307 known TFs out of $\sim 6000$ distinct proteins participate in (currently estimated) $\sim 2 \times 10^{5}$ regulatory associations [2]. Yet, these are still not sufficiently well characterized to tell whether an arbitrarily chosen TF up- or down-regulates a target gene in a given context. Therefore, the structural optimality -in the above sense- of the yeast's regulatory network as a whole is not possible to assess with the currently available information.

Nevertheless, the small subnetwork responsible for the cell-cycle in yeast (YCC) is very well studied [18, 35 38]. A handful of regulatory proteins or complexes that drive the cell division and the interactions between them have been identified. Furthermore, several models of regulatory dynamics involving these proteins have been shown to yield the experimentally observed expression profiles [18, 37, 38]. We will consider two such models here, and demonstrate their high degree of coherence as a proof of concept.

Once the cell reaches a critical size [39, 40], the cell division is initiated. It starts from the $G_{1}$ phase of the mother cell and ends in the $G_{1}$ phases of the mother and daughter cells. The two models we consider here reproduce in proper order all the intermediate expression stages that the cell traverses during division, via the time-evolution equations given in Table I. They feature the same set of key TFs/complexes (vertices of the network) that drive DNA replication and the accompanying cell division in yeast. These models are depicted in Fig. 2, where blue nodes indicate auto-repressor elements $\left(c_{i i}=-1\right)$ and two-colored (blue+magenta) nodes represent multiprotein complexes. The model proposed by Li et al. [18] in Fig. $2 \mathrm{a}$ is, to our knowledge, the first successful implementation of Boolean dynamics to yeast's cell cycle and has been widely used in later studies. We will refer to this model as $M 1$ from now on. More recently, Mangla et al. improved $M 1$ by introducing additional interactions and varying interaction weights, while at the 


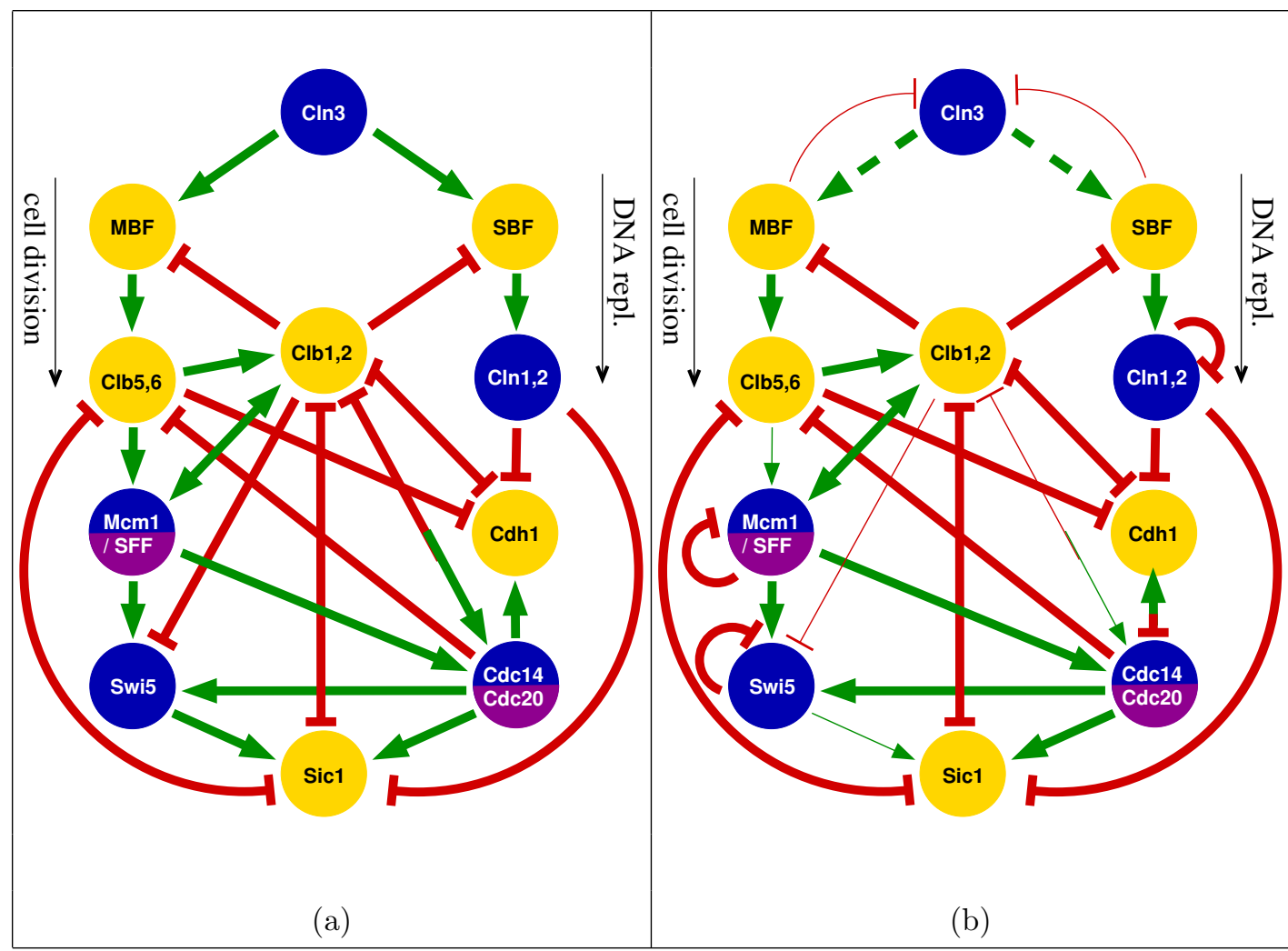

FIG. 2: Two model systems for yeast cell cycle. (a) Model $M 1$ by Li et al. (b) Model $M 2$ by Hong et al. The weights for interactions in $M 2$ are 1, 1/3 and 3 for normal, thin and dashed lines, respectively.

same time extending the Boolean formalism to allow some of the vertices to have three different states $(0,1,2)$ [37]. This work was later refined by Hong et al. [38] in order to capture crucial checkpoint conditions overlooked in $M 1$. We will refer to the YCC model of Hong et al. (shown in Fig. 2 $\mathrm{b}$ ) as $M 2$ from now on.

Time-evolution equations for $M 1$ and $M 2$ are given in Table If. Here, $\sigma_{i}(t)$ is the expression state which takes the value '1' when the node $i$ is active and ' 0 ' otherwise, except for Swi5 and Clb2 which can also take the value of '2' (strong expression) in M2. The interactions considered in $M 1$ (Fig. 22 ) differ only by their sign, i.e., $c_{i j} \in\{-1,0,1\}$, while for $M 2 c_{i j} \in\{0, \pm 1 / 3, \pm 1, \pm 3\}$ (shown with different edge thicknesses in Fig. 2b). $\theta_{i, 1}$ and $\theta_{i, 2}$ are threshold values that trigger the state shifts $0 \leftrightarrow 1$ and $1 \leftrightarrow 2$, respectively. While reproducing the time-evolution of $M 2$ given in Ref. [38, we concluded that the observed dynamics and basin sizes are reproducible only if an additional parameter, $\delta$, that introduces a hysteresis to up/down regulation events is added to the evolution dynamics (see Table II). This crucial detail appears to be accidentally omitted in the reference article. We give the numerical values of the parameters above in Table $\mathbb{I}$ and refer the 
reader to the original references for further details of the two models.

\begin{tabular}{|c|c|c|}
\hline \multirow{2}{*}{$\sigma_{i}(t+d t)$} & \multicolumn{2}{|c|}{ if } \\
\cline { 2 - 3 }$=$ & with $\theta_{i, 1}=0$ & Mith $\theta_{i, 1}=0.5$ and $\theta_{i, 2}=1.5$ \\
\hline \multirow{2}{*}{$\sigma_{i}(t)-1$} & or $\quad \sum_{j \neq i} c_{i j} \sigma_{j}(t)<\theta_{i, \sigma_{i}(t)}$ & \\
& $\sum_{j \neq i} c_{i j} \sigma_{j}(t)=\theta_{i, \sigma_{i}(t)}$ and $c_{i i}=-1$ & $\sum_{j} c_{i j} \sigma_{j}(t)<\theta_{i, \sigma_{i}(t)}-\delta$ \\
\hline$\sigma_{i}(t)+1$ & $\sum_{j \neq i} c_{i j} \sigma_{j}(t)>\theta_{i, \sigma_{i}(t)+1}$ & $\sum_{j} c_{i j} \sigma_{j}(t) \geq \theta_{i, \sigma_{i}(t)+1}$ \\
\hline$\sigma_{i}(t)$ & otherwise & otherwise \\
\hline
\end{tabular}

TABLE I: Time evolution equations for the two models ( $M 1$ and $M 2)$ discussed in the text. $\delta=0.75$ (a parameter that appears to be omitted in the original reference) was found to be consistent with the basin sizes in 38 .

While a deterministic implementation of the above equations follows the experimentally resolved expression dynamics, a stochastic version will occasionally end up in different attractors listed in Table II. The biological relevance of such relatively low probability states is unclear [18, 41]. Nevertheless, the degree of coherence supported by the structure of the regulatory network under the dynamical evolution given in Table 1 should to be defined through all of its attractors. This also facilitates a fair comparison of the YCC network with randomized ensembles for which a classification of attractors according to their biological relevance is meaningless. On the other hand, we do present numerical results obtained through basin-size weighted averaging, which, in the case of YCC, corresponds to essentially focusing on the $G_{1}$ attractor. Note also that, the trivial fixed point $\boldsymbol{\sigma}=0$, which is common to all networks considered in this study, is left out in Table II as well as in our analysis below.

Also given in Table $\Pi$ are the coefficients of coherence for the attractors of $M 1$ and $M 2$, calculated using equation in Table If. For the whole network, we obtain $\alpha_{c}=0.97(M 1)$ and 0.89 (M2) with uniformly weighted attractors and $0.99(M 1)$ and 0.98 (M2) with basin-size weighted averaging. We observe that, all the common attractors of $M 1$ and $M 2$ (including the biologically relevant $G_{1}$ phase) are either fully coherent or almost so.

Is the YCC network optimized towards maximal coherence? A meaningful assessment of the above numbers is possible only in the background of corresponding results on properly selected 


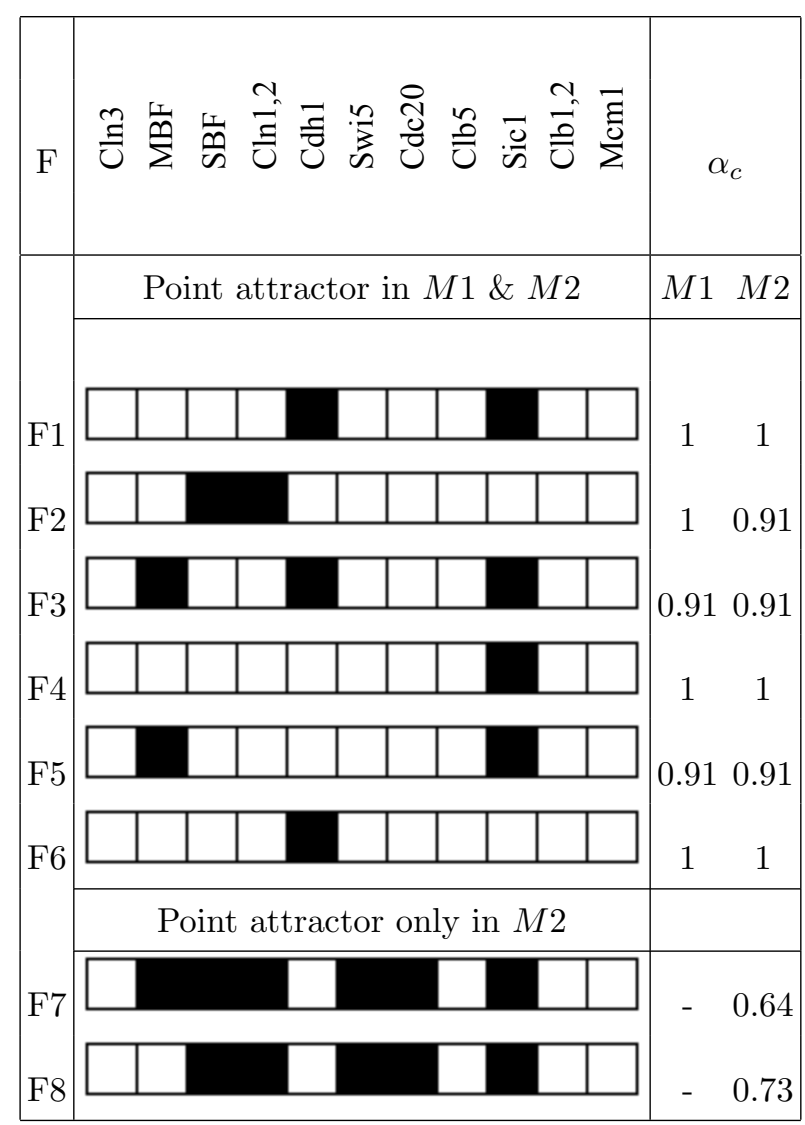

TABLE II: YCC attractors under the dynamics given in Table I and their coherence, $\alpha_{c}$. Black boxes indicate the active genes and the first row corresponds to the experimentally verified $G_{1}$ phase.

random ensembles (for a similar analysis to determine frequent network motifs in biological networks see, for example, Refs. [11, 42].) Therefore we next perform an analysis on directed graphs of the same size with similar characteristics. We do this in two different ways, as explained below.

\section{B. Coherence: yeast vs random networks}

Even a network composed of random associations, each gene up- or down-regulating an arbitrary subset of genes, will display a nonzero degree of coherence determined by the laws of statistics. Two obvious factors that contribute to $\alpha_{c}$ are: the number of regulating partners per node, $k$, and the fraction of up-regulating interactions in the network, $p$. (See section III C.) While we will investigate the dependence of $\bar{\alpha}_{c}$ (avarage of $\alpha_{c}$ values over an ensemble of networks) on $k$ and $p$ below, it is sensible to compare yeast's cell-cycle network against the appropriate random ensemble with $(k, p)=\left(k_{y c c}, p_{y c c}\right)$. Excluding self loops, model $M 1$ above has 14 inhibitory and 15 activatory interactions, that is, $\left(k_{y c c}, p_{y c c}\right)_{1} \simeq(2.63,0.52)$. Similarly, model $M 2$ has 20 inhibitory and 15 


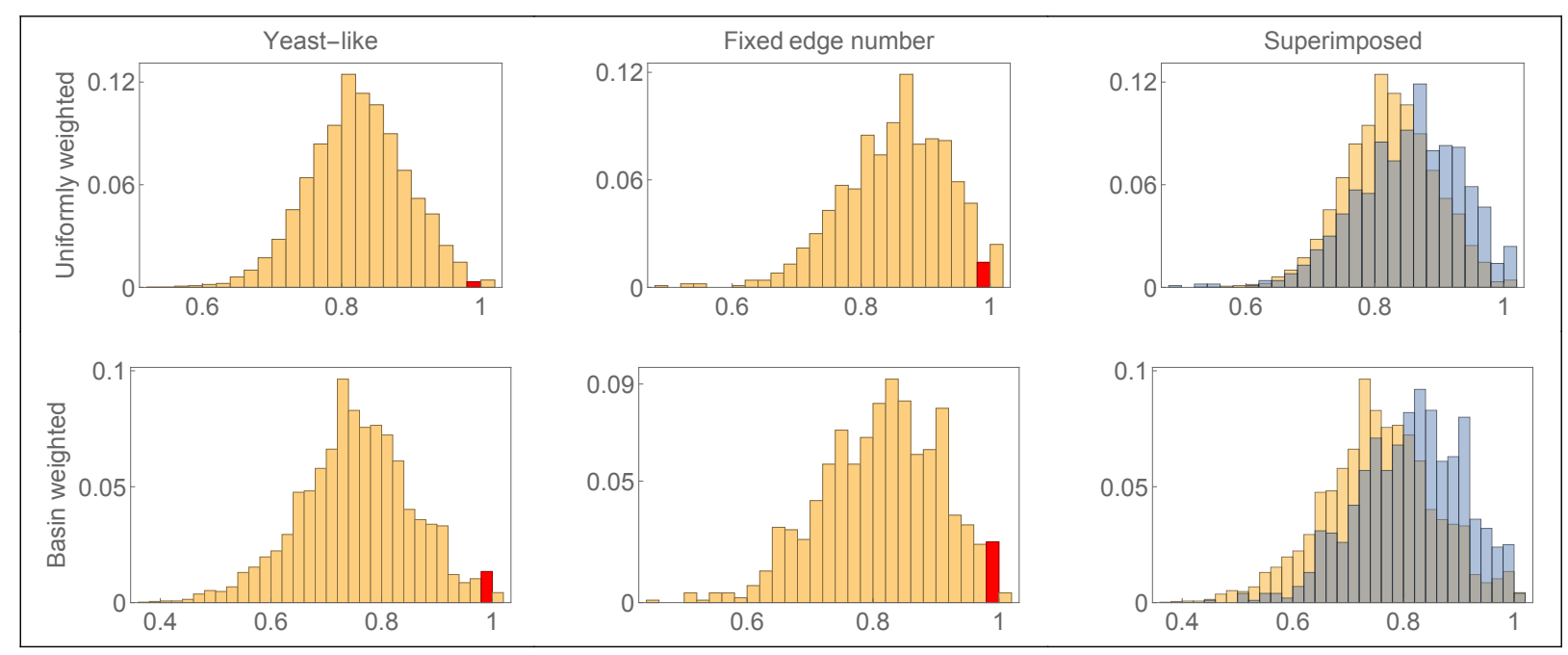

FIG. 3: The histograms for $\alpha_{c}$ calculated for "YCC-like" networks (first column) and the random $\left(N_{y c c}, k_{y c c}, p_{y c c}\right)$ ensemble (second column) generated by using model $M 1$ described in the text. The third column shows the superposition of the two histograms for comparison. $\alpha_{c}$ for the yeast's cell-cycle model is shown in red everywhere.

activatory interactions, yielding $\left(k_{y c c}, p_{y c c}\right)_{2} \simeq(3.18,0.43)$.

In order to construct an ensemble of networks with fixed $(N, k, p)$, we first generated connected, undirected random graphs with $N$ regulatory elements (nodes), and $N k$ interactions (edges) between them ( $k \geq 1$ is required for connectivity.) Then, each edge was assigned one of the two possible interaction types (activator or repressor), such that a fraction $p$ of them up-regulate, and the rest down-regulate their targets. Note that, a node may act as an activator for one target and as a repressor for another. This is in line with the behavior observed in YCC, as well as recent research which suggests that the TFs involved in both kinds of regulation may be more common than previously thought [43, 44]. We compared models $M 1$ and $M 2$ with the corresponding random ensembles R1 and R2 generated using network parameters $\left(N_{y c c}, k_{y c c}, p_{y c c}\right)_{1,2}$, respectively.

We also applied a more stringent test of coherence in the chosen YCC models by comparing them with more "yeast-like" ensembles. These ensembles (Y1 and Y2) were generated by forcing each node to preserve its number of incoming and outgoing edges, separately for each type of interaction. This was achieved by a simple edge-shuffling procedure that switches the targets of two randomly selected edges in the network, subject to the condition that the edges are of the same type. In all the random ensembles considered, we also separately fixed the fraction of the auto-repressor nodes $\left(c_{i i}=-1\right)$ to the corresponding values $5 / 11(M 1)$ and 3/11 (M2), for a fair comparison. 


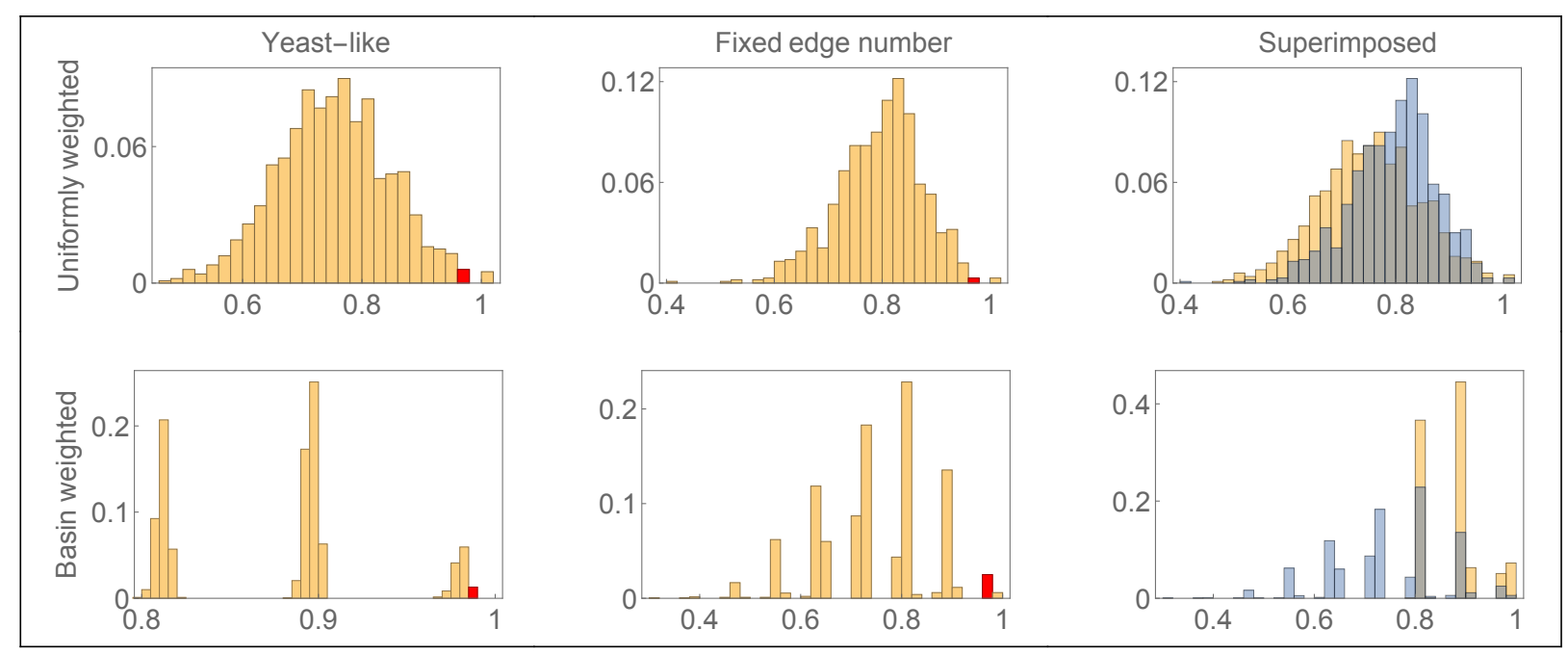

FIG. 4: The histograms for $\alpha_{c}$ calculated for "YCC-like" networks (first column) and the random $\left(N_{y c c}, k_{y c c}, p_{y c c}\right)$ ensemble (second column) generated by using model $M 2$ described in the text. The third column shows the superposition of the two histograms for comparison. $\alpha_{c}$ for the yeast's cell-cycle model is shown in red everywhere.

Overall, two sets of $10^{4}$ random networks $(R 1, Y 1)$ and $(R 2, Y 2)$ were generated for each YCC model. The probability distributions of $\alpha_{c}$ obtained from each ensemble are shown in Fig. 3 and Fig. 4 separately for $M 1$ and $M 2$, together with the value obtained from the actual models for comparison.

Next, we compared the $\alpha_{c}$ distibutions above with those from the "yeast-like" ensembles Y1 and Y2, which preserve the particular set of in-/out-degree pairs for the two interaction types at each node of the model networks $M 1$ and $M 2$. Surprisingly, Y1 (both with and without basinsize weighted averaging) and Y2 (without basin-size weighted averaging) yield a reduced degree of coherence when compared with the respective ensembles $\mathrm{R} 1$ and $\mathrm{R} 2$, as is evident from Fig. 3 . The corresponding mean values along with standard deviations for $\bar{\alpha}_{c}$ are listed in Table III. We found that the coherence values of the actual networks lie in the top $4 \%$ in all of the random ensembles R1, R2, Y1 and Y2. Based on these observations, we conclude that the exceptionally high level of coherent regulatory activity observed in the two YCC models is not solely due to by the particular local decoration of the networks' nodes. 


\begin{tabular}{|c|c|c|c|c|c|c|c|}
\hline \multicolumn{2}{|c|}{} & R1 & Y1 & M1 & R2 & Y2 & M2 \\
\hline \multirow{2}{*}{$\bar{\alpha}_{c} \pm \sigma$} & Uniformly weighted & $0.85 \pm 0.08$ & $0.82 \pm 0.07$ & 0.97 & $0.79 \pm 0.07$ & $0.75 \pm 0.09$ & 0.89 \\
\cline { 2 - 9 } & Basin weighted & $0.81 \pm 0.09$ & $0.75 \pm 0.10$ & 0.99 & $0.75 \pm 0.11$ & $0.87 \pm 0.06$ & 0.98 \\
\hline
\end{tabular}

TABLE III: Mean and standart deviation values for $\alpha_{c}$ of random ensembles along with the $\alpha_{c}$ values of model networks $M 1$ and $M 2$.

\section{Coherence vs network parameters}

In order to investigate the dependence of mean degree of coherence on $k$ and $p$, we next considered (for simplicity of implementation) only the YCC model $M 1$ above. We, again, generated $10^{4}$ distinct, random networks of size $N=11$ (as in YCC), now with $k \in[1.6,5]$ and $p=0.5 \simeq p_{y c c}$, as well as with $k=k_{y c c}=29 / 11$ and $p \in[0,1]$. Possible isomorphism of the ensemble members was ruled out by ensuring that each network in the ensemble has a different eigenvalue spectrum. We also generated much larger networks with $N=110$ nodes (corresponding to a 10 -fold increase in size) for comparison and with $k$ and $p$ values in the same intervals as above, in order to check the network size dependence of our results. Then the attractors of the dynamics given in Table $\mathbb{1}$ were found for each network by an exhaustive search for $N=11$ and by sampling $2^{11}$ initial states for $N=110$. Finally, $\alpha_{c}$ were calculated separately for each network over corresponding attractors of the dynamical evolution described by Eq. (2). The dependence of the ensemble mean $\bar{\alpha}_{c}$ on $p$ and $k$ is shown in Figs. 5(a)\&5(b) respectively.

$\bar{\alpha}_{c}$ changes only slightly with the number of nodes $N$ for a given $(k, p)$ pair: increasing the network size 10-fold yields a similar behavior, with $<9.8 \%$ difference in the worst case (Fig. 5). Therefore, despite the smallness of the model system considered here, our results on random ensembles may be expected to serve as a reasonable null-hypothesis for coherence in the global regulatory network of yeast and other organisms.

It may at first sight be surprising that $\bar{\alpha}_{c}$ is asymmetric with respect to $p=1 / 2$, given that the number of genes with incoming edges that both up- and down-regulate the gene is maximized precisely at this point. The observed asymmetry is due to the fact that the fraction of active nodes at the fixed points is a monotonically increasing function of $p$ (the insets of Fig. 5). Close to $p=0$ and $p=1$, most genes are regulated in the same direction by their regulating partners, hence an increase in coherence is observed in these extremes. While the fraction of genes with conflicting incoming edges peaks at $p=1 / 2$, the minimum of $\bar{\alpha}_{c}$ is shifted towards larger $p$ values (more up-regulation), where the chances are higher for antagonistic regulatory partners of a gene to be 


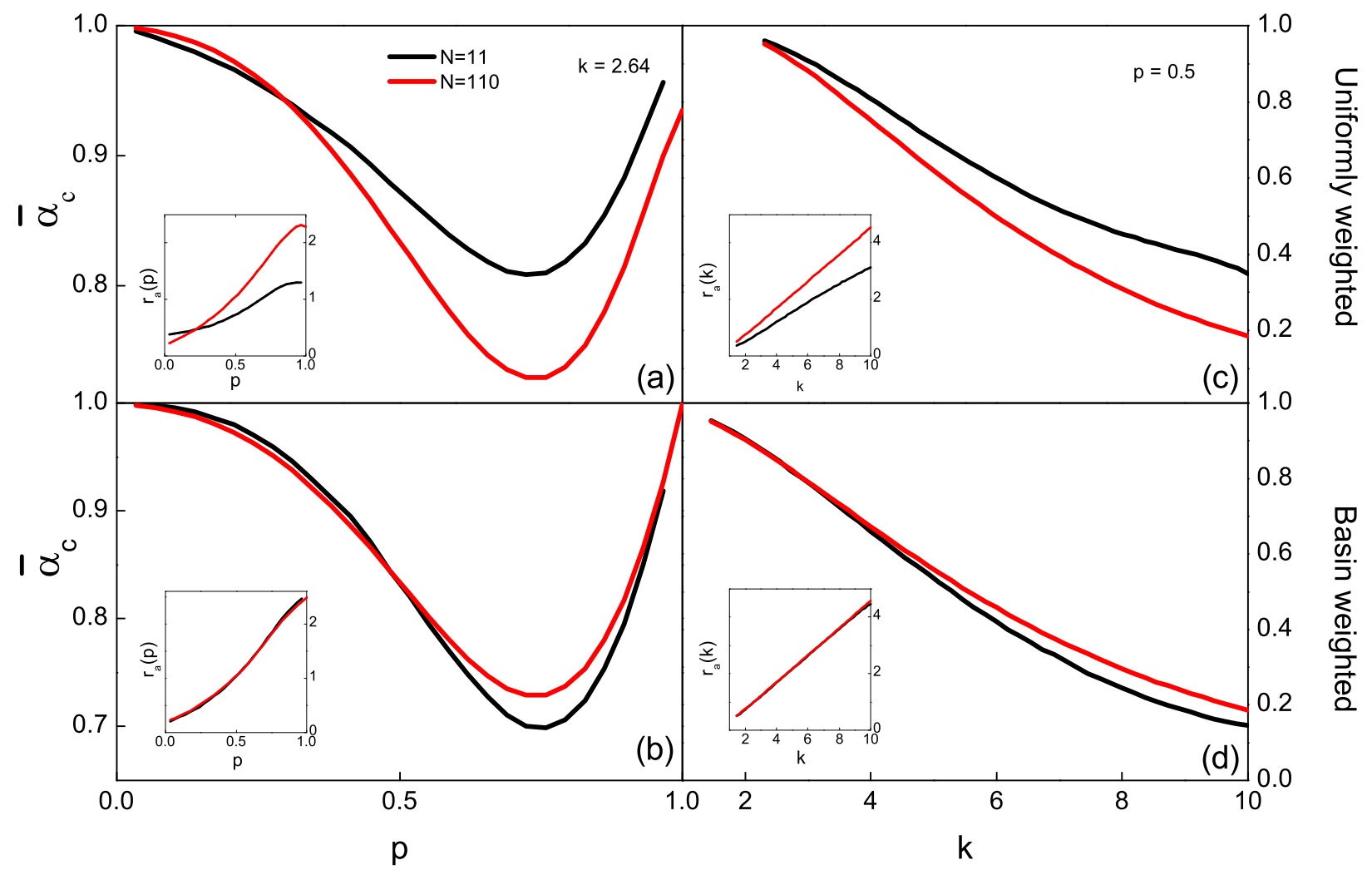

FIG. 5: (a,b) Degree of coherence, $\bar{\alpha}_{c} v s . \quad p$ for $k=k_{y c c}=29 / 11$ with and without consideration of the basin sizes. (c,d) Degree of coherence, $\bar{\alpha}_{c} v s . k$ for $p=0.5 \simeq p_{y c c}$ with and without consideration of the basin sizes, respectively. The insets show the average fraction of the regulating active nodes at the fixed points.

simultaneously expressed.

A simple analytical model based on the above reasoning can be shown to reproduce the observed behavior. Having shown the weak dependence of our results on the network size, let us consider the large- $N$ limit. For fixed $(k, p)$, define the average number of active regulating partners per node be $r_{a}(k, p)$. The data from the ensembles with $N=11$ and $N=110$ suggest the form below (see Fig. 5(a,b) inset):

$$
r_{a}(k, p)=r_{\min }+p^{\gamma}\left(r_{\max }-r_{\min }\right)
$$

where $r_{\min , \max }$ are $k$-dependent. Note that, a typical attractor of a network with all inhibitory interactions $(p=0)$ will still have a few nodes "on" in absence of active down-regulating partners, hence $r_{\min }>0$. When $p=1$, we expect most of the nodes to be turned on. A node is coherently regulated, if all its active regulating partners act unanimously. $\alpha_{c}$ measures essentially the probability for this event across the network's attractors. Treating the states of neighbors regulating a 


\begin{tabular}{|c|c|c|c|c|c|c|c|}
\cline { 3 - 8 } \multicolumn{2}{c|}{} & \multicolumn{3}{c|}{ for $r_{a}$} & \multicolumn{3}{c|}{ for $\bar{\alpha}_{c}$} \\
\cline { 3 - 8 } \multicolumn{2}{c|}{} & $r_{\text {min }}$ & $r_{\text {max }}$ & $\gamma$ & $r_{\min }$ & $r_{\text {max }}$ & $\gamma$ \\
\hline \multirow{2}{*}{$\mathrm{N}=11$} & no basin & 0.36 & 1.46 & 1.49 & 1.03 & 1.66 & 1.91 \\
\cline { 2 - 8 } & with basin & 0.19 & 2.69 & 1.54 & 1.02 & 2.55 & 2.64 \\
\hline \multirow{2}{*}{$\mathrm{N}=110$} & no basin & 0.21 & 2.55 & 1.44 & 1.03 & 2.26 & 2.42 \\
\cline { 2 - 8 } & with basin & 0.22 & 2.60 & 1.49 & 1.03 & 2.23 & 2.34 \\
\hline
\end{tabular}

TABLE IV: Fit values for the model.

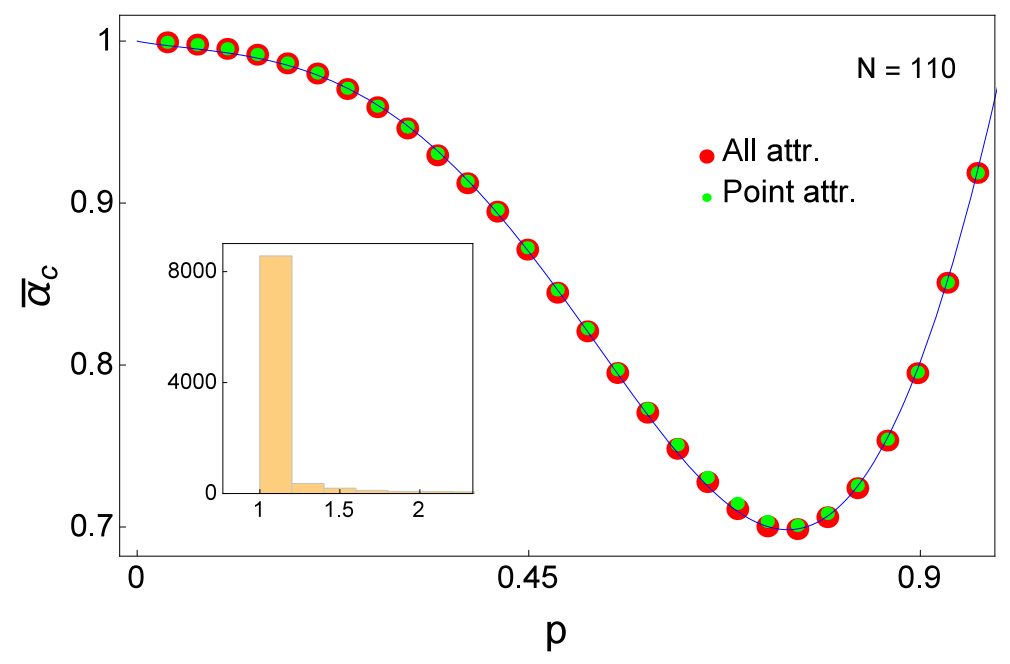

FIG. 6: The contribution of cyclic attractors for $N=110$ to basin-size weighted values of $\bar{\alpha}_{c}$. The dependence of $\bar{\alpha}_{c}$ on $p$ is calculated over all attractors (red) and over point attractors only (green), for comparison. The blue curve is a fit calculated by using equations $(78 \times 8$. The average cycle length distribution for $p=0.5$ given in the inset is typical of all the ensembles considered here, irrespective of the averaging method used.

given node as independent random variables, this probability can be expressed as

$$
\bar{\alpha}_{c}(p, k)=p^{r_{a}}+(1-p)^{r_{a}}
$$

The functional form in equation (7) yields a good fit for both $r_{a}$ and $\bar{\alpha}_{c}$ with $\left(r_{\min }, r_{\max }, \gamma\right)$ values listed in Table IV. The agreement between the basin-averaged $\bar{\alpha}_{c}$ vs $p$ for $N=110$ and the analytical form above is shown in Fig. 6. On the other hand, the parameter values yielding the best fit to $\bar{\alpha}_{c}$ and $r_{a}$ differ. This discrepancy may be attributed to the nonuniformity of the in-degree distribution and the contribution of correlations, both of which are not captured by the simple treatment above.

The monotonic decrease of $\bar{\alpha}_{c}$ with increasing $k$, the mean number of regulating partners per node, shown in Fig. 5(c,d) is hardly surprising. It reflects the fact that the genes with fewer 
active regulators are less likely to receive conflicting inputs. The number of active neighbors per node when $p=0.5 \simeq p_{y c c}$ increases almost linearly with $k$, suggesting that the overall number of simultaneously active nodes remains a constant, essentially determined by $p$.

The results presented here are identical to the naked eye when non-point-attractors (with period $>1$ ) are excluded from the calculation of $\alpha_{c}$ on random networks. Such cycles appear relatively infrequently with the given regulatory dynamics (see Fig. 6 inset). Therefore, the fact that the yeast's networks in (Fig. II) has only point attractors does not seem to be relevant to its extreme degree of coherence, either. Investigation of structural features of coherent networks (such as local motif statistics, directed cycles, etc.) is planned as a future study.

\section{DISCUSSION}

We defined a measure for the degree of coherence in gene regulatory networks. Using the proposed measure, we showed that the cell-cycle network of the budding yeast displays exceptionally high degree of harmonious regulatory activity, which is in line with earlier observations on its robustness. The proposed measure of coherence sets the YCC network aside, within an ensemble of networks of the same size and composition. We found that, achieving coherent regulation is most difficult in systems when roughly $25 \%$ of the interactions are repressory. While this ratio is about $1 / 2$ for the yeast's cell-cycle network, YCC yields a degree of coherence which is significantly higher than those of random networks with identical network parameters such as size, average degree, and +/- interaction ratio. When basin basin sizes are ignored (uniform weighting), optimality of the YCC network is even more pronounced within the ensemble of yeast-like networks that preserve the in-/out-degrees of nodes separately for each interaction type. A deeper analysis of highly coherent random networks is necessary to pinpoint the structural determinants of these unique and possibly biologically relevant class of networks.

Perhaps the most far-reaching question inspired by our findings is whether coherence in gene regulation is a prevalent motif across organisms in nature. In order to check this, the presented analysis needs to be extended to the cell-cycle regulation networks of other organisms (for example the fission yeast [45]), as well as GRNs responsible for different biological processes (examples in the literature include cell differentiation and segmentation networks [46 48]). Such an investigation is currently in progress and will shed light on the generality of our observations on the cell-cycle network of the budding yeast. An affirmative answer to the question above would point to the interesting possibility of a Hebbian-like selection mechanism for regulatory interactions. That is 
because, the coherence property defined in this work can be seen as a generalization of the Hebb's rule for neural networks [], which is usually stated as "neurons that fire together, wire together". A mechanism of selection for coherent networks would similarly promote positive regulation (and demote negative regulation) among genes that are expressed simultaneously, and the vice versa for an "on" gene regulating an "off" gene. Rapid evolution of regulatory interactions as found in recent studies [49] resonates with this scenario, for it provides the fertile ground on which such a mechanism can effectively produce coherent networks in short evolutionary time scales.

\section{Acknowledgements}

A.K. is grateful for the hospitality of M. Kardar during a visit to MIT and a stimulating discussion with the rest of the biophysics group there. We also thank D. Yuret and C. Dunn of Koç University for beneficial exchanges.

\section{References}

[1] T.-H. Yang, C.-C. Wang, Y.-C. Wang, and W.-S. Wu, Database 2014, bau014 (2014).

[2] M. C. Teixeira, P. T. Monteiro, J. F. Guerreiro, J. P. Gonçalves, N. P. Mira, S. C. dos Santos, T. R. Cabrito, M. Palma, C. Costa, A. P. Francisco, et al., Nucleic acids research 42, D161 (2014).

[3] U. Güldener, M. Münsterkötter, G. Kastenmüller, N. Strack, J. van Helden, C. Lemer, J. Richelles, S. J. Wodak, J. García-Martínez, J. E. Pérez-Ortín, et al., Nucleic acids research 33, D364 (2005).

[4] R. Münch, K. Hiller, H. Barg, D. Heldt, S. Linz, E. Wingender, and D. Jahn, Nucleic acids research 31, 266 (2003).

[5] R. A. Drysdale, M. A. Crosby, et al., Nucleic acids research 33, D390 (2005).

[6] A. Chatr-aryamontri, B.-J. Breitkreutz, S. Heinicke, L. Boucher, A. Winter, C. Stark, J. Nixon, L. Ramage, N. Kolas, L. ODonnell, et al., Nucleic acids research 41, D816 (2013).

[7] K. Klemm and S. Bornholdt, Proceedings of the National Academy of Sciences of the United States of America 102, 18414 (2005).

[8] S. Mangan and U. Alon, Proceedings of the National Academy of Sciences 100, 11980 (2003).

[9] D. Thieffry, Briefings in bioinformatics 8, 220 (2007).

[10] J. J. Tyson and B. Novák, Annual review of physical chemistry 61, 219 (2010).

[11] U. Alon, An introduction to systems biology: design principles of biological circuits (CRC press, 2006).

[12] V. Sevim and P. A. Rikvold, Journal of theoretical biology 253, 323 (2008). 
[13] O. Cinquin and J. Demongeot, Comptes rendus biologies 325, 1085 (2002).

[14] R. Sansom, The British Journal for the Philosophy of Science 59, 169 (2008).

[15] Z. Yuan, C. Zhao, Z. Di, W.-X. Wang, and Y.-C. Lai, Nature communications 4 (2013).

[16] G. Yan, J. Ren, Y.-C. Lai, C.-H. Lai, and B. Li, Physical review letters 108, 218703 (2012).

[17] S. Wuchty, Proceedings of the National Academy of Sciences p. 201311231 (2014).

[18] F. Li, T. Long, Y. Lu, Q. Ouyang, and C. Tang, Proceedings of the National Academy of Sciences of the United States of America 101, 4781 (2004).

[19] X. Zhang, K. Jantama, J. C. Moore, L. R. Jarboe, K. T. Shanmugam, and L. O. Ingram, Proceedings of the National Academy of Sciences 106, 20180 (2009).

[20] J. E. Niven and S. B. Laughlin, Journal of Experimental Biology 211, 1792 (2008).

[21] S. B. Laughlin, R. R. d. R. van Steveninck, and J. C. Anderson, Nature neuroscience 1, 36 (1998).

[22] Z. Eelderink-Chen, G. Mazzotta, M. Sturre, J. Bosman, T. Roenneberg, and M. Merrow, Proceedings of the National Academy of Sciences 107, 2043 (2010).

[23] O. E. Akman, S. Watterson, A. Parton, N. Binns, A. J. Millar, and P. Ghazal, Journal of The Royal Society Interface 9, 2365 (2012).

[24] M. I. Stefan and N. Le Novère, PLoS computational biology 9, e1003106 (2013).

[25] M. Thattai and A. Van Oudenaarden, Proceedings of the National Academy of Sciences 98, 8614 (2001).

[26] P. S. Swain, M. B. Elowitz, and E. D. Siggia, Proceedings of the National Academy of Sciences 99, 12795 (2002).

[27] J. Paulsson, Nature 427, 415 (2004).

[28] N. T. Ingolia, PLoS biology 2, e123 (2004).

[29] K. Kaneko, PLoS One 2, e434 (2007).

[30] Y. Zhang, M. Qian, Q. Ouyang, M. Deng, F. Li, and C. Tang, Physica D: Nonlinear Phenomena 219, 35 (2006).

[31] A. Fauré, A. Naldi, C. Chaouiya, and D. Thieffry, Bioinformatics 22, e124 (2006).

[32] K. Kaizu, S. Ghosh, Y. Matsuoka, H. Moriya, Y. Shimizu-Yoshida, and H. Kitano, Molecular systems biology 6 (2010).

[33] P. E. Hodges, A. H. McKee, B. P. Davis, W. E. Payne, and J. I. Garrels, Nucleic Acids Research 27, 69 (1999).

[34] G. D. Bader, A. Heilbut, B. Andrews, M. Tyers, T. Hughes, and C. Boone, Trends in cell biology 13, $344(2003)$.

[35] W.-B. Lee and J.-Y. Huang, FEBS letters 583, 927 (2009).

[36] Y. Okabe and M. Sasai, Biophysical journal 93, 3451 (2007).

[37] K. Mangla, D. L. Dill, and M. A. Horowitz, PloS one 5, e8906 (2010).

[38] C. Hong, M. Lee, D. Kim, D. Kim, K.-H. Cho, and I. Shin, BMC systems biology 6, 129 (2012).

[39] G. Johnston, J. Pringle, and L. Hartwell, Experimental cell research 105, 79 (1977). 
[40] I. Rupeš, Trends in Genetics 18, 479 (2002).

[41] R. Albert and H. G. Othmer, Journal of theoretical biology 223, 1 (2003).

[42] R. Milo, S. Shen-Orr, S. Itzkovitz, N. Kashtan, D. Chklovskii, and U. Alon, Science Signalling 298, 824 (2002).

[43] M. J. Hickman and F. Winston, Molecular and cellular biology 27, 7414 (2007).

[44] M. Ptashne, Nature chemical biology 7, 484 (2011).

[45] M. I. Davidich and S. Bornholdt, PLoS One 3, e1672 (2008).

[46] E. Remy and P. Ruet, Bioinformatics 24, i220 (2008).

[47] L. Mendoza, D. Thieffry, and E. R. Alvarez-Buylla, Bioinformatics 15, 593 (1999).

[48] J. Krumsiek, C. Marr, T. Schroeder, and F. J. Theis, PloS one 6, e22649 (2011).

[49] D. Schmidt, M. D. Wilson, B. Ballester, P. C. Schwalie, G. D. Brown, A. Marshall, C. Kutter, S. Watt, C. P. Martinez-Jimenez, S. Mackay, et al., Science 328, 1036 (2010). 